\title{
Depredación de arañas hacia visitantes florales y herbívoros, balance entre mutualismo y antagonismo
}

\author{
Mariana TadeY ${ }^{\bowtie, 1}$; Roger Ayazo ${ }^{2}$; Farah Carrasco-Rueda ${ }^{3}$; Yuliana Christopher ${ }^{4}$; \\ Marisol Domínguez ${ }^{5}$; Giomara La Quay-VelázqueZ ${ }^{6} \&$ Miriam San José $^{7}$
}

1. CONICET, Laboratorio Ecotono, CRUB - Universidad Nacional del Comahue, San Carlos de Bariloche, Argentina. 2. Instituto de Ecología A.C., Veracruz, México. 3. Center for Conservation Education and Sustainability, Smithsonian Conservation Biology Institute, National Zoological Park, Washington, USA. 4. Centro de Biodiversidad y Descubrimiento de Drogas. Instituto de Investigaciones Científicas y Servicios de Alta Tecnología, Ciudad del Saber, Panamá. 5. Laboratorio de Ecología y Comportamiento Animal, Departamento de Ecología, Genética y Evolución, Facultad de Ciencias Exactas y Naturales, Universidad de Buenos Aires, Argentina. 6. Departamento de Biología, Universidad de Puerto Rico, San Juan, USA. 7. Laboratorio de Ecología de Paisajes Fragmentados, Centro de Investigaciones en Ecosistemas, Universidad Nacional Autónoma de México.

\begin{abstract}
RESUMEN. Las relaciones entre arañas y plantas pueden ser complejas y afectar de modos múltiples a sus plantas hospederas. La araña verde, Peucetia viridans, utiliza el camuflaje para cazar visitantes florales e insectos herbívoros por lo cual podría reducir tanto los niveles de herbivoría como los de reproducción de la planta hospedadora. Además, esta araña suele estar asociada a plantas con tricomas que les facilitan la caza de sus presas. Determinamos el balance de esta doble interacción en una hierba (Ruellia nudi ora) en un Bosque seco de Costa Rica. En tres sitios de muestreo realizamos un experimento en el cual cambiamos el color de la araña pintándola de rojo para determinar si un incremento en su detectabilidad afectaba la visita de polinizadores. Estimamos la abundancia de arañas, polinizadores e insectos herbívoros así como también los niveles de herbivoría, densidad de tricomas y producción de frutos/flor de la planta. La presencia de la araña no estuvo asociada a cambios en la tasa de visita de polinizadores ni a una menor fructificación. Las arañas estuvieron asociadas a plantas con menor densidad de tricomas. Los niveles de herbivoría fueron mayores en plantas con arañas que sin arañas. Sin embargo, las plantas con menor nivel de herbivoría y sin arañas presentaron mayor densidad de tricomas. Los sitios mostraron diferencias en el ensamble de herbívoros y polinizadores pero esto no afectó las interacciones estudiadas. Este estudio es el primero en mostrar que las arañas están asociadas a una densidad baja de tricomas. Si las arañas realmente depredaran significativamente los visitantes florales, el valor adaptativo de los tricomas sería doble; por un lado la reducción de la herbivoría por insectos y por otro repeler la presencia de un eventual depredador de sus visitantes florales. Estos resultados resaltan la importancia de estudiar en forma conjunta las interacciones mutualistas y antagonistas para determinar su importancia relativa.
\end{abstract}

[Palabras clave: densidad de tricomas, frecuencia de visitas, producción de frutos, presión por depredación]

\begin{abstract}
AвSTRACт. Spider predation on floral visitors and herbivores, balance between mutualism and antagonism: Spiders-plant interactions are usually complex and affect their host plants in multiple ways. The lynx spider Peucetia viridans camouflages to hunt flower visitors and herbivores potentially reducing the levels of herbivory and reproduction of their host plant. Plus, these green spiders are usually associated with plant species presenting trichomes, which usually facilitate the spider predation. We determined the balance of this double interaction on the forb Ruellia nudi ora in a dry forest of Costa Rica. In three different sites we performed an experiment changing the spider color to red to determine whether the increment on the spider detectability affects pollinators visitation frequency. We also estimated spider, pollinators and herbivores abundance and the levels of herbivory, trichomes density and fruit set. The presence of the spider was not associated to a decrease in pollinators visitation or fruit set. Spiders were associated to plants with low trichomes density. Herbivory was higher in plants with spiders than in plant without them, however, plants with low herbivory level and without spiders presented higher trichomes density. Sites differed in their assemblages of pollinators and herbivores but this did not affect the interactions studied. This is the first study showing that the spider $P$. viridans is associated with plants of the same species with low trichome density. If this spider affects pollinators visitations, the adaptive value of trichomes would be double; they reduce herbivory and repel the presence of pollinators predators. These results highlight the relevance of studying both mutualistic and antagonistic interactions to determine their relative importance.
\end{abstract}

[Keywords: trichome density, visitation frequency, fruit set, predation risk]

\section{INTRODUCCIÓN}

La polinización por insectos es un proceso fundamental para la reproducción de muchas plantas. Sin embargo, la visita de insectos a las flores - parte fundamental de este procesodepende de numerosos factores. Variaciones en la abundancia, distribución y recompensa

Editora Asociada: Marina Omacini

$\bowtie$ mtadey@conicet.gov.ar 
de los recursos (e. g., flores, néctar) afectan la tasa de visita de los polinizadores (Kunin 1992; Stang et al. 2006; Ebeling et al. 2008). Sin embargo, esta tasa de visita también puede depender del riesgo de depredación afectando indirectamente la reproducción de las plantas (Romero et al. 2011) si bien este factor ha sido relativamente menos estudiados que aquellos relacionados con la disponibilidad de recursos (Jones \& Dornhaus 2011; Romero et al. 2011).

Las arañas constituyen uno de los principales depredadores de insectos voladores (Oxford \& Gillespie 1998). Particularmente, muchas especies cazadoras representan un serio riesgo para los insectos polinizadores, ya que se camuflan en las flores $u$ hojas cercanas y los atrapan durante las visitas a las flores (Stevens \& Merilaita 2009; Abbott 2010). Se ha documentado que esta presión de depredación generada por las arañas sobre los visitantes florales puede afectar su comportamiento (Jones \& Dornhaus 2011) disminuyendo la polinización y la producción de frutos (Higginson et al. 2010). Sin embargo, los resultados encontrados no son consistentes (Dukas 2005; Dukas \& Morse 2005; Arango et al. 2012).

Las arañas verdes (Peucetia spp.) suelen vivir asociadas a especies de plantas que poseen tricomas glandulares (VasconcellosNeto 2006). Se cree que esta asociación puede ser mutualista dado que la araña presenta mimetismo con la planta y se alimenta de los herbívoros que se acercan en busca de alimento. Sin embargo, la dieta de estas arañas es generalista e incluye potenciales polinizadores (Nyffeler et al. 1992). La planta puede obtener un beneficio de la araña si ésta disminuye su nivel de herbivoría (Romero et al. 2008) pero también podría perjudicarse si la araña se alimenta de sus polinizadores (Arango et al. 2012).

En Bosques tropicales de Centroamérica es relativamente común encontrar arañas cazadoras camufladas en plantas para capturar a visitantes florales (Janzen 1991). Esta compleja interacción es muy frecuente en los Bosques secos estacionales de Costa Rica. El objetivo de esta investigación es estudiar el sistema conformado por la araña verde Peucetia viridans y la planta Ruellia nudiflora, una herbácea comúnmente asociada a zonas disturbadas (Ortegón-Campos et al. 2012). Para ello, nos planteamos los siguientes objetivos particulares que llevamos a cabo en el parque nacional Palo Verde: (1) documentar la interacción entre la araña $P$. viridans y los visitantes florales en relación a la estrategia de camuflaje de las arañas, su efecto sobre el comportamiento de los polinizadores y las consecuencias sobre las plantas y (2) determinar el impacto de la araña sobre los niveles de herbivoría y su asociación con los tricomas, facilitadores de captura. Nuestra primer hipótesis establece que si las arañas utilizan la estrategia de camuflaje (i. e., cripsis) para maximizar la captura de sus presas, esperamos que, por un lado, sus colores sean similares al sustrato adonde se encuentran $y$, por otro lado, que arañas coloreadas experimentalmente tengan una menor tasa de captura que las no coloreadas. Nuestra segunda hipótesis propone que si los visitantes polinizadores pueden detectar a las arañas y modifican su comportamiento, esperamos menor cantidad/tiempo de visitas a flores con arañas comparadas con aquellas sin arañas. A su vez, si las arañas disminuyen los niveles de herbivoría de $R$. nudiflora al alimentarse de los visitantes herbívoros, esperamos que la herbivoría de las plantas con arañas sea menor que las plantas sin arañas. Finalmente, si la araña aprovecha los tricomas glandulares para cazar esperamos que el número de arañas y tricomas estén positivamente asociados.

\section{MATERIALES y MÉTODOS}

\section{Área de estudio, experimento y muestreos}

El área de estudio se localizó dentro del Parque Nacional Palo Verde, en la Estación Biológica Palo Verde manejada por la Organización para Estudios Tropicales (OET), Costa Rica. Este parque incluye una de las áreas de Bosque seco mejor conservadas en Centroamérica (Janzen 1991), con zonas de Bosque caducifolio y vegetación siempre-verde asociada a cursos de aguas (Bosques en galería), áreas de pastizales que eran antiguas fincas ganaderas y un humedal. La precipitación es muy estacional alcanzando los $1600 \mathrm{~mm}$ entre junio y noviembre mientras que entre enero y mayo es nula o muy escasa (Janzen 1991).

Se seleccionaron distintos sectores dentro del área de estudio, en donde la especie vegetal en estudio, Ruellia nudiflora era muy frecuente. Esta especie es una herbácea perenne, auto-compatible generalmente asociada a disturbios (OrtegónCampos et al. 2012). Posee un sistema reproductivo mixto con dos tipos de flores, cleistógamas y casmógamas, las primeras se auto-polinizan obligatoriamente ya que no abren, mientras que las segundas aunque también poseen niveles altos de auto-fertilización experimentan visitas de polinizadores (Munguía-Rosas et al. 2012). Las hojas son ovales y están dispuestas en pares y toda la planta está cubierta de tricomas glandulosos. 
Algunos herbívoros frecuentes en $R$. nudiflora son larvas de Lepidoptera (e. g. Anartia jatrophae), algunos coleópteros y grillos (Ortegón-Campos et al. 2012). Además, diversos insectos visitan las flores de esta planta como moscas, avispas, abejas y mariposas de diversos grupos taxonómicos.

Peucetia viridans (Oxyopidae) es una especie de araña cazadora (i. e., que no construye tela) de color verde claro (Willey \& Adler 1989). P. viridans posee una dieta generalista consumiendo insectos de tamaño muy variable (Willey \& Adler 1989). El tamaño del adulto puede llegar a los $22 \mathrm{~mm}$ en hembras y $12 \mathrm{~mm}$ los machos. En el área de estudio se la encuentra frecuentemente asociada $R$. nudiflora (Acanthaceae), una herbácea de color similar al de la araña (Información Suplementaria, Figura S1).

El estudio se realizó durante febrero de 2013, época seca y período de mayor actividad de polinizadores en el Parque Nacional Palo Verde. Elegimos tres sitios diferentes, uno en el borde el humedal (Humedal), otro cercano al humedal pero sin Bosque (Sol) y el último sitio de Bosque aun más alejado del humedal (Bosque). Además de las diferentes características estos sitios poseen distinta intensidad de uso e impacto del ganado (alta, media y baja, respectivamente) manejado por el Parque Nacional. Esta frecuencia de impacto fue estimada en relación al tiempo que el ganado está presente en cada sector.

Para determinar si la araña es mimética marcamos plantas que ya poseían arañas y plantas sin arañas en dos de los sitios (Sol y Humedal). El número de plantas sin arañas (control) fue de 20 para Humedal y 10 para Sol. Las plantas con arañas recibieron tres tratamientos: 1) marcamos en el abdomen arañas con color rojo (contrastante con su color natural) $\left.\left(\mathrm{N}_{\text {Humedal }}=20 ; \mathrm{N}_{\mathrm{Sol}}=11\right) ; 2\right)$ arañas pintadas con verde como un control del marcaje $\left(\mathrm{N}_{\text {Humedal }}=4\right)$; y 3) y arañas sin ningún tratamiento $\left(\mathrm{N}_{\text {Humedal }}=66\right.$; $\mathrm{N}_{\mathrm{Sol}}=24$ ). Se muestrearon simultáneamente ambos sitios para evitar efectos del día de muestreo. Se eligieron estos dos sitios para el experimento porque su mutua cercanía permitía muestrear en simultáneo. Se monitoreó a lo largo del día el éxito de captura de las arañas contabilizando los cadáveres en las plantas como una medida indirecta de la dieta de la araña.

Un estudio previo en el mismo sitio de estudio probó que el color de la araña es similar al de la planta hospedadora mediante la comparación de composición aditiva promedio de colores (composición aditiva de rojo, verde y azul), la saturación promedio de rojo, verde y azul, de cada araña y el de su entorno utilizando el programa ImageJ $(n=30 ; p=0.34)$, (Vega López, J.V. ; datos sin publicar). Consecuentemente, no realizaremos medidas adicionales sobre la coloración de la araña y su entorno.

Para evaluar el efecto de la araña sobre el comportamiento de visitantes florales se realizaron períodos de observación de $10 \mathrm{~min}$. a lo largo de todo el día (en total se realizaron 120 períodos de observación). Se determinó el número e identidad de visitantes florales por flor, y el número de flores visitadas. Estas observaciones se realizaron en la plantas marcadas con los cuatro tratamientos anteriormente descriptos y de manera simultánea en los mismos dos sitios.

Para evaluar el efecto de la araña sobre la herbivoría de la planta hospedadora y el papel de los tricomas en este proceso en las plantas marcadas se tomaron tres fotos de distintas partes de la planta de manera estandarizada (i. e. las fotos fueron tomadas a una distancia de $20 \mathrm{~cm}$ ) en donde estimamos la herbivoría como el número de hojas con herbivoría sobre el total de hojas observadas de cada fotografía. También contabilizamos el número de tricomas en un $\mathrm{mm}^{2}$ bajo lupa en 5 hojas al azar de cada planta marcada (i.e. $\mathrm{N}=84$ ).

Para determinar la adecuación de las plantas en presencia/ausencia de la araña colectamos al azar una infrutescencia por planta (68 plantas en total, 27 sin araña y 41 con arañas) a las cuales se contabilizó el número de frutos y flores totales ya que se podían cuantificar aquellas flores que no habían producido fruto. En cada planta se observó, previo a la colecta de infrutescencias, si había arañas o mudas en ellas. En estas mismas plantas se estimó también la densidad de tricomas $/ \mathrm{mm}^{2}$ en tres hojas seleccionadas al azar.

Por otro lado, se midió la abundancia de polinizadores en cada sitio mediante transectas de $150 \mathrm{~m} \times 2 \mathrm{~m}$ de ancho durante $15 \mathrm{~min}$. de observación, en las cuales se caminaba lentamente y se anotaba todo insecto, sea herbívoro o polinizador, que se observaba y su ubicación (16 transectas totales: 8 Bosque, 4 Sol, 4 Humedal). Los insectos se clasificaron por morfo-especies como una estimación de la riqueza. Además, realizamos una medida de la abundancia de las arañas y de los insectos herbívoros presentes en cada sitio en parcelas de $50 \mathrm{~cm} \times 50 \mathrm{~cm}$ (44 parcelas totales: 12 Bosque, 22 Sol, 10 Humedal). De esta manera también obtuvimos una medida adicional de la abundancia de herbívoros en los sitios. En estas parcelas se midieron también el tamaño de la araña y la altura a la que se encontraba $(\mathrm{cm})$ ubicada en la planta.

\section{Análisis estadístico}

Para evaluar si el comportamiento de los polinizadores variaba según la visibilidad de la araña, comparamos el número de visitas y el de flores visitadas entre plantas con arañas sin pintar, arañas pintadas de rojo, de verde y sin arañas. Para esto realizamos un ANCOVA de dos factores (sitio y tratamiento), en donde el número de flores de la planta y su altura fueron consideradas como covariables.

Para comparar la proporción de frutos/flor y el número de tricomas $/ \mathrm{mm}^{2}$ en hojas de plantas con y sin araña, realizamos un ANOVA de una vía. Por otro lado, para determinar si la herbivoría era diferente entre plantas con y sin arañas se realizó 
una prueba $t$ Student. También se realizó una correlación no paramétrica de Spearman entre la herbivoría y la densidad de tricomas y entre el número de arañas y la densidad de tricomas. Además realizamos un ANOVA de dos vías (sitio y presencia de araña como factores) para comparar proporción de herbivoría y la densidad de tricomas de las plantas, entre los sitios con presencia/ ausencia de araña.

Para determinar el efecto del sitio sobre la interacción entre la araña, herbívoros y visitantes florales comparamos entre los diferentes sitios las siguientes variables repuesta empleando ANOVAs de una vía: el número de arañas/parcela, altura en la que se encontraba la araña $(\mathrm{cm})$, su tamaño (variable categórica; grande-mediano-pequeño) y el número de flores/ parcela. Las arañas de tamaño grande eran aquellas que median aproximadamente $20 \mathrm{~mm}$, las medianas medían entre $10-15 \mathrm{~mm}$ y las pequeñas eran menores a $7 \mathrm{~mm}$. Asimismo, analizamos el efecto del sitio sobre la abundancia de polinizadores y herbívoros obtenidos por el método de transectas mediante curvas de rarefacción. Las variables respuestas obtenidas fueron: el número de individuos, número de especies (morfoespecies), número de gremios (i. e., polinizadores, herbívoros y otros), número de morfo-especies de polinizadores y número de morfo-especies de herbívoros para cada transecta.

Aquellas variables que no cumplían con los supuestos de normalidad y homogeneidad de varianzas fueron transformadas. Las variables índice de herbivoría, densidad de tricomas y altura de las plantas fueron transformadas a su logaritmo natural, y la proporción frutos/flor se transformó con arcoseno de la raíz cuadrada.

\section{Resultados}

\section{Efecto de la araña}

El número de visitas de polinizadores y de flores visitadas, corregido por la cantidad flores en las plantas y su altura, fue similar entre plantas con arañas pintadas, sin pintar $\mathrm{y}$ plantas $\sin$ arañas ( $\mathrm{p}$ polinizadores $=$ 0.44 , p flores $=0.6)$ así como entre sitios ( $p$ polinizadores $=0.68$, $\mathrm{p}$ flores $=0.95$; Figura $1 \mathrm{~A}, \mathrm{~B})$. Ambas co-variables tuvieron un efecto positivo y significativo pero diferente sobre las variables respuesta. El número de flores/ planta afectó positivamente al número de flores visitadas $\left(\mathrm{F}_{1,143}=3.76, \mathrm{p}=0.05,14.4 \pm\right.$ 1.9 , media \pm ES de la co-variable ) mientras que la altura de la planta tuvo un efecto positivo sobre la número de visitas $\left(\mathrm{F}_{1,143}=\right.$ $18.5, \mathrm{p}=0.00003,37.8 \pm 1.2 \mathrm{~cm}$, media $\pm \mathrm{ES}$ de la covariable). Además encontramos una interacción entre el sitio y los tratamientos significativa para el número de visitas $\left(\mathrm{F}_{2,143}\right.$ $=3.8, \mathrm{p}=0.025)$ pero no significativa para el
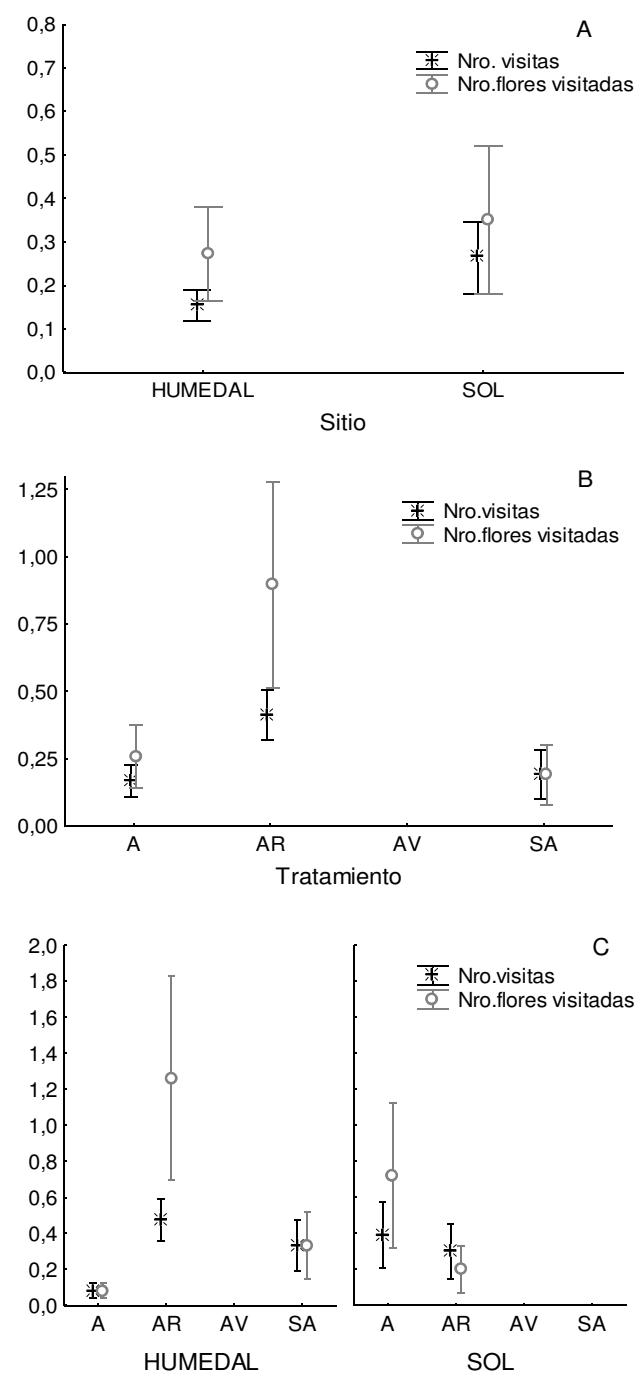

Figura 1. A) Número de polinizadores que visitan las flores de Ruellia nudiflora y número de flores visitadas/ visita en los dos sitios estudiados (Humedal y Sol). B) Número de polinizadores que visitan las flores de $R$. nudiflora y número de flores visitadas/visita de acuerdo al tratamiento aplicado a Peucetia viridans. A = Araña sin pintar; $\mathrm{AR}=$ Araña con marca roja; $\mathrm{AV}=$ Araña con marca verde y $\mathrm{SA}=\mathrm{Sin}$ araña. C) Número de polinizadores que visitan las flores de $R$. nudiflora y número de flores visitadas/visita por sitio y tratamiento. Los valores representan el promedio \pm 1 ES.

Figure 1. A) Number of visitors on Ruellia nudiflora and the number of flowers visited/visit in each studied site (Humedal $=$ wetland and Sol $=$ prairie). B) Number of visitors on R. nudiflora and the number of flowers visited/visit according to the treatment applied on the spider Peucetia viridans. See details in legend in Spanish.

número de flores visitadas $(\mathrm{p}=0.17$, ver Figura 1C). Las plantas con arañas marcadas de rojo presentaron un mayor número de visitas en Humedal. En cambio en Sol el tratamiento "araña sin pintar" fue el que presentó mayor número de flores visitadas respecto al resto aunque similar tasa de visitas. 
El promedio de la proporción frutos/flor de plantas con y sin arañas fue similar $(p=0.81$, Figura 2A). Por el contrario, encontramos que la densidad de tricomas en esas mismas plantas con y sin araña fue significativamente diferente $\left(\mathrm{F}_{1,67}=4.77, \mathrm{p}=0.03\right.$, Figura $\left.3 \mathrm{~B}\right)$, ya que las arañas estaban en plantas con menor densidad de tricomas.

El nivel de herbivoría se correlacionó positivamente con el número de arañas $\left(\mathrm{r}_{\mathrm{s}}=\right.$ $0.25, \mathrm{n}=84, \mathrm{p}=0.04)$ pero no con el número de tricomas $\left(\mathrm{r}_{\mathrm{s}}=-0.01, \mathrm{n}=84, \mathrm{p}=0.9\right)$. Acorde al primer resultado, el promedio de herbivoría fue mayor en plantas con araña que sin araña $(\mathrm{t}=-2.02, \mathrm{n}=94, \mathrm{p}=0.04)$. Por otra parte, tampoco encontramos una relación entre el número de arañas y la densidad de tricomas $(\mathrm{r}=0.04, \mathrm{n}=84, \mathrm{p}=0.6)$. Al analizar los niveles de herbivoría y la densidad de tricomas en las hojas teniendo en cuenta el sitio y la presencia de araña no encontramos efectos significativos $\left(\mathrm{p}_{\text {sitio }}=0.83, \mathrm{p}_{\text {araña }}=0.35, \mathrm{p}_{\text {interacción }}=0.82\right.$; Figura $4 \mathrm{~A}, \mathrm{~B})$.
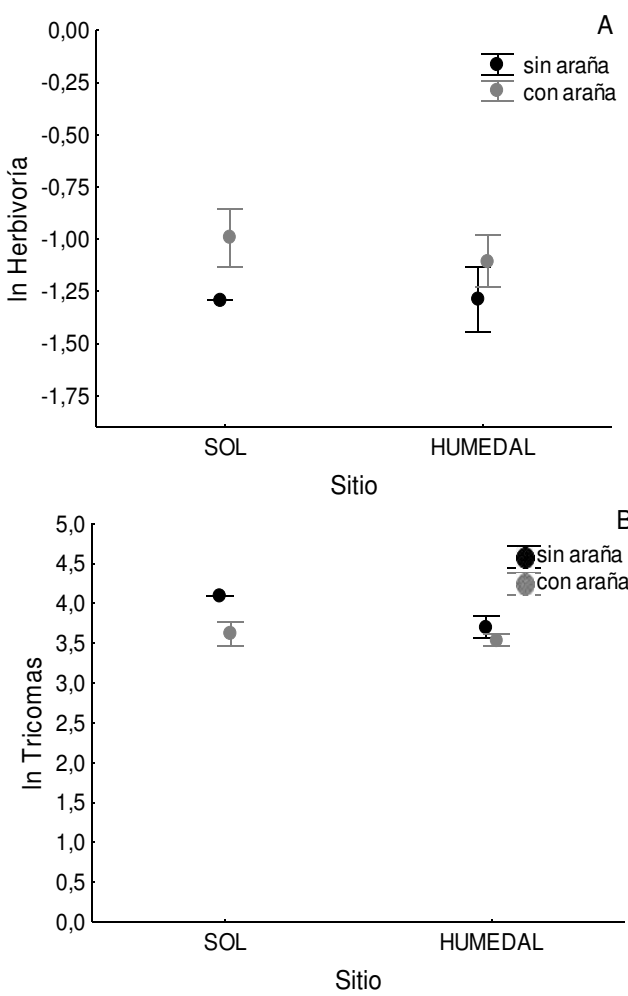

Figura 2. Promedio ( \pm 1 ES) de la proporción de frutos/flor (A) y el número de tricomas $/ \mathrm{mm}^{2}$ de hojas (B), de Ruellia nudiflora en relación a la presencia (1) o ausencia (0) de la araña Peucetia viridans ( ${ }^{*}$ diferencias significativas, $\mathrm{p}<$ 0.05).

Figure 2. Mean $( \pm 1 \mathrm{SE})$ fruit set $(\mathrm{A})$ and number of trichomes $/ \mathrm{mm}^{2}$ in the leaves (B) of Ruellia nudiflora in relation to the presence (1) or absence (0) of the spider Peucetia viridans $\left({ }^{*}\right.$ significant diferences, $\left.\mathrm{p}<0.05\right)$.
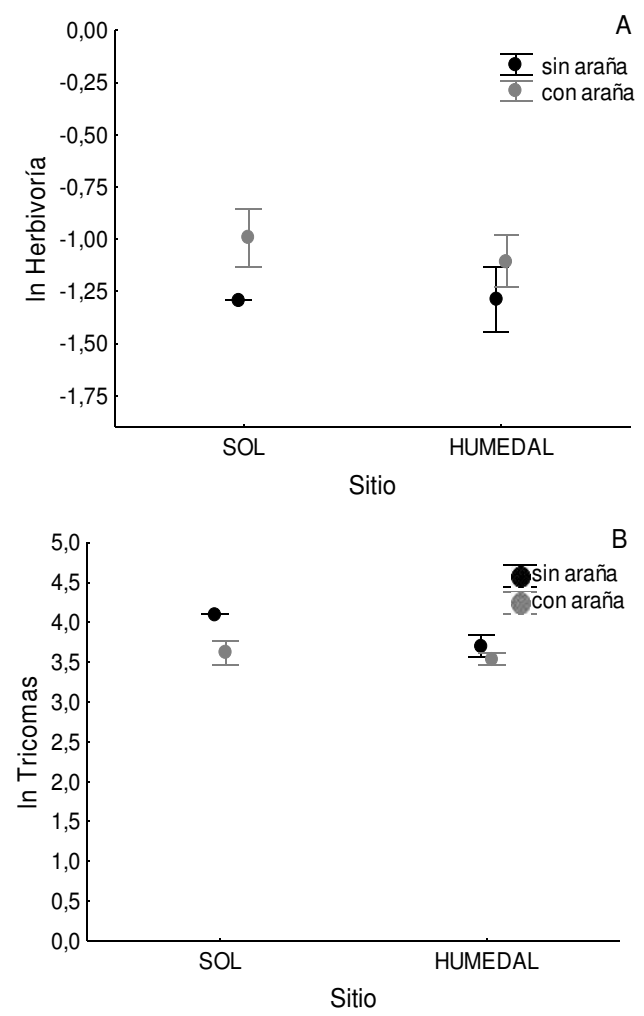

Figura 3. Promedio ( \pm 1 ES) del logaritmo natural (ln) del Indice de herbivoría (A) y del número de tricomas/ mm2 (B) en las hojas de Ruellia nudiflora en relación a la presencia o ausencia de la araña Peucetia viridans y sitios muestredos (Sol y Humedal).

Figure 3. Mean $( \pm 1$ SE) of the natural logarithm (ln) of herbivory index $(\mathrm{A})$ and the number of trichomes $/ \mathrm{mm} 2$ (B) in Ruellia nudiflora leaves according the presence or absence of Peucetia viridans in the study sites (Sol= prairie and Humedal= wetland).

Sólo presenciamos 4 eventos de depredación a lo largo de $1200 \mathrm{~min}$. de observación (2 en Sol $\mathrm{y}$ dos en Humedal), dos de los cuales fueron realizados por las arañas pintadas de rojo. Los insectos capturados en estos episodios fueron una oruga, una abeja (Apis mellifera), un grillo y un díptero.

\section{Efectos del sitio}

Los diferentes sitios fueron similares en cuanto a la abundancia de arañas $(p=0.65)$. Sin embargo, el tamaño de la araña y la altura a la que se la encontró, al igual que el número de flores / parcela variaron entre sitios $\left(\mathrm{F}_{2,30}=\right.$ $3.99, \mathrm{p}=0.02 ; \mathrm{F}_{2,44}=32, \mathrm{p}=0.0001 ; \mathrm{F}_{2,46}=3.2, \mathrm{p}=$ 0.04 , respectivamente; Figura $4 \mathrm{~A}, \mathrm{~B})$. El sitio Bosque presentó en promedio mayor número de flores que el resto, mientras que el tamaño de las arañas fue mayor en Humedal, y Sol presentó plantas de menor altura que el resto de los sitios. 

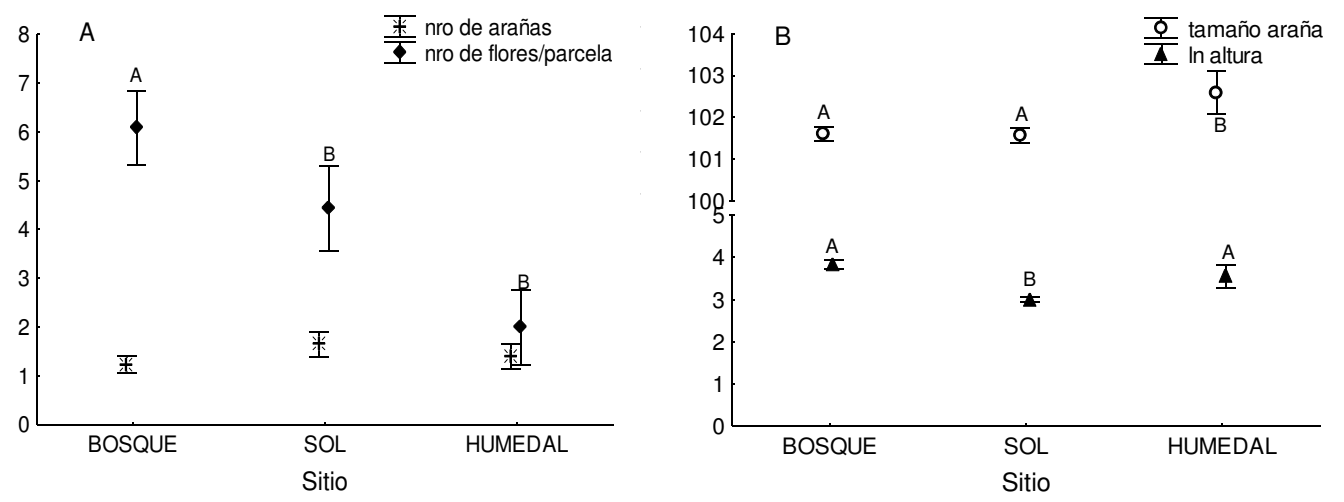

Figura 4. Promedio ( \pm 1 ES) del número de arañas, de flores/parcela (A), tamaño de la araña y altura donde fue encontrada la araña (B) en cada uno de los sitios muestreados (Bosque, Sol y Humedal). Las letras mayúsculas diferentes denotan diferencias estadísticas.

Figure 4. Mean ( \pm 1 SE) number of spiders, flowers / plot (A), spider size and height where spiders were found (B) in the plants in each study sites $($ Bosque $=$ forest, $\mathrm{Sol}=$ prairie and Humedal= wetland). Capital letters denote statistical diferences.
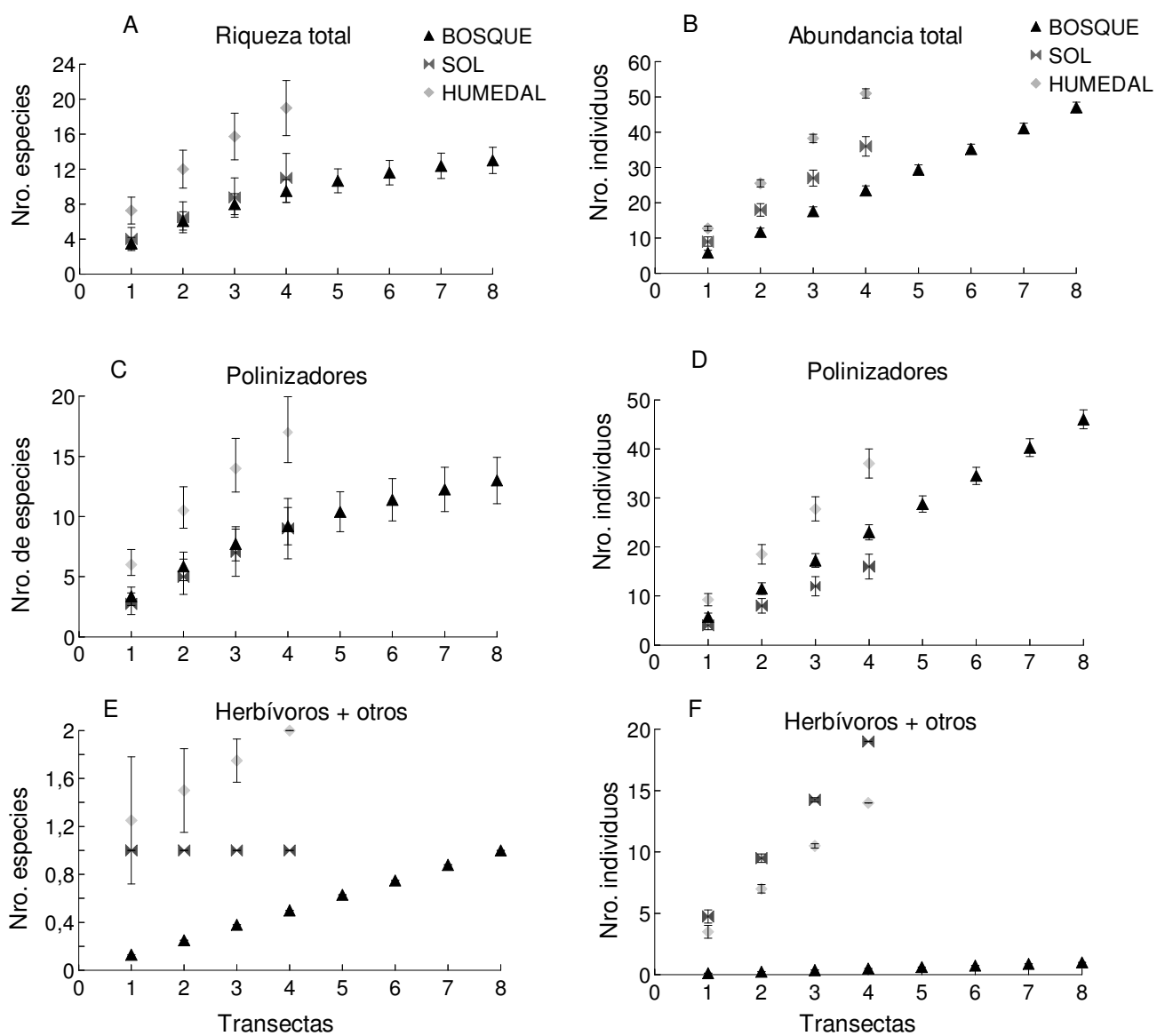

Figura 5. Curvas de rarefacción para los tres sitios de muestreo (Bosque, Sol y Humedal) graficadas por el número de transectas realizadas: A) número total de morfo-especies esperados; B) número de individuos totales; C) número de morfo-especies de polinizadores; D) número de individuos de polinizadores; E) El número de morfo-especies del gremio de herbívoros y otros; F) El número de individuos del gremio de herbívoros y otros. Las barras de error representan los desvíos estándar.

Figure 5. Rarefaction curves for each of the study sites (Bosque $=$ forest, $\mathrm{Sol}=$ prairie and Humedal= wetland). Expected number of morpho-species (A) and total individuals (B) graphed by the number of transects. Number of pollinators morpho-species (C) and individuals (D). Number of morpho-species (E) and indiviudals (F) of herbivores and other groups. Error bars denote standard deviation. 
Las curvas de rarefacción muestran que el sitio de mayor riqueza y abundancia general fue Humedal, mientras que Bosque y Sol mostraron riquezas y abundancias similares. No obstante, ninguna de las curvas se estabiliza, lo que significa que se requiere un mayor esfuerzo de muestreo para determinar la riqueza y abundancia de esos sitios. Si se compara a igual número de transecta $(\mathrm{N}=4)$ encontramos que el número de morfo-especies es menor en el Bosque, intermedio en Sol y mayor en Humedal (Figura 5A). lo mismo ocurre con el número de individuos (Figura 5B) y en el gremio de los polinizadores donde la riqueza fue mayor en Humedal y similar en los otros dos sitios (Figura 5C), aunque su abundancia fue mayor en Humedal, intermedia en Bosque y menor en Sol (Figura 5D). En cuanto al gremio de herbívoros y otros gremios que fueron evaluados conjuntamente, observamos que la riqueza era similar en Sol y Humedal y bastante menor en Bosque (Figura 5E,F), aunque estas comparaciones no son estadísticas y por lo tanto no son concluyentes.

\section{DisCUSIÓN}

En este estudio pusimos a prueba distintas hipótesis para determinar si la araña $P$. viridans influye sobre la adecuación de la planta $R$. nudiflora. En primer lugar, analizamos si las arañas utilizan el camuflaje para maximizar la captura de los visitantes florales de $R$. nudiflora. En segundo lugar, analizamos si la presencia de arañas, mediante la depredación de herbívoros, afecta el nivel de herbivoría. Paralelamente, estudiamos si la araña utiliza los tricomas de la planta para cazar sus presas (herbívoros o polinizadores). Y en último lugar se determinó si los distintos sitios estudiados afectaron alguno de estos procesos.

Con respecto a la primera hipótesis encontramos evidencia a favor de que la araña parece mimética para los polinizadores ya que estos visitaron con similar frecuencia plantas con y sin arañas. Sin embargo, el tratamiento de araña pintada de rojo presentó mayor número de visitas. Esto podría deberse a que en esas plantas había mayor número de flores comparado con el resto de las plantas estudiadas, un sesgo involuntario, no manejado, de nuestro experimento. Adicionalmente, la similar tasa de visitas de polinizadores a plantas con y sin arañas, y la mayor tasa de visitas a plantas con más flores independientemente de una mayor visibilidad de la araña- sugieren que la disponibilidad de recursos alimenticios es más importante para los polinizadores a la hora de seleccionar un sitio de forrajeo que la presencia de la araña. Esto es opuesto a lo sugerido en trabajos anteriores que proponen que el riesgo de depredación o señales de depredación previa desalientan la visita de polinizadores (Dukas 2005; Abbott 2006; Brechbühl et al. 2010; Jones \& Dornhaus 2011; Romero et al. 2011) . No obstante, los eventos de depredación fueron menos frecuentes que lo esperado a partir de publicaciones previas (Arango et al. 2000, 2012).

Por otro lado, la pintura roja en la araña $P$. viridans, no afectó la cantidad de visitas de polinizadores como si lo hizo en trabajos anteriores (Arango et al. 2012). Esto también podría deberse a que el color rojo utilizado pudo no haber sido tan evidente para los polinizadores de $R$. nudiflora y por lo tanto, no haya cambiado considerablemente el mimetismo de la araña. Trabajos anteriores, en el mismo sitio, en donde la araña fue coloreada con azul (simulando las flores de R. nudiflora) obtuvieron los mismos resultados (Vega López, J. V.; datos sin publicar). No obstante, en otras especies se ha observado un cambio en el comportamiento de los polinizadores luego de la aplicación de pintura roja como también ante arañas modelos (Louda 1982; Romero et al. 2008). De todas maneras, la forma en que estos cambios influyen en la frecuencia de visitas sobre la adecuación de la planta pueden variar según la disponibilidad de recursos alimenticios de la araña (e.g. abundancia tanto de polinizadores como de herbívoros) (Arango et al. 2012).

En el sitio de estudio no encontramos evidencia de un efecto de la presencia de la araña sobre la adecuación de la planta (i. e., frutos/flor). Estos resultados concuerdan con que la tasa de visitas fue similar entre plantas con y sin arañas. Sin embargo, la medida de adecuación tomada en este estudio fue una estimación poco precisa. Resulta necesario profundizar sobre este aspecto ya que esta especie vegetal es auto-compatible y no necesita obligatoriamente de polinizadores para su reproducción (Munguía-Rosas et al. 2012) .

En cuanto a la hipótesis de que la araña se alimenta de herbívoros y por lo tanto disminuye el nivel de herbivoría de las plantas hospedadoras, encontramos lo opuesto a lo esperado. Plantas con mayor número de arañas presentaron un mayor nivel de herbivoría. En otros estudios, la presencia 
de arañas del género Peucetia no afectó los niveles de herbivoría de la planta hospedera sino sólo de algunos grupos de herbívoros (Morais-Filho \& Romero 2008). Sin embargo, el patrón encontrado en este estudio puede explicarse por el hecho de que las plantas que tuvieron mayor nivel de herbivoría y abundancia de arañas tenían, en promedio, menor densidad de tricomas (ver Figura $4 \mathrm{~A}, \mathrm{~B})$. En otras palabras, las arañas estarían eligiendo plantas con menor densidad de tricomas, lo que a su vez estaría relacionado con un mayor nivel de daño foliar. El hecho de que la araña está asociada a plantas con menor densidad de tricomas (ver Figura 2B), y que las plantas sin arañas tiene menor herbivoría sugiere que los tricomas son una eficiente estrategia anti-herbívoros en esta especie de planta (Hare \& Elle 2002). Sin embargo, esto parece contradecir lo encontrado en estudios anteriores que sugieren que la araña podría utilizar los tricomas de la planta para poder atrapar las presas que quedan pegadas (Vasconcellos-Neto 2006, Romero et al. 2008b, Morais-Filho \& Romero 2010). Posiblemente, una elevada densidad de tricomas le dificulte a la araña cazar a sus presas. Se ha observado que en plantas con alta densidad de tricomas hay una importante cantidad de detritos provenientes de otras plantas, polvo, frutos y semillas pegados a los tallos y hojas (M. Tadey, observación personal). Todo esto podría ser un obstáculo para el desplazamiento de la araña dentro de la planta, reducir el número de visitantes florales $\mathrm{y} / \mathrm{o}$ disminuir la probabilidad de que los insectos que frecuentan R. nudiflora se peguen. Esto podría explicar por qué las arañas se asociaron mayormente a plantas con menor densidad de tricomas a pesar de que aquellas poseían un mayor número de flores. En este sentido, este es el primer estudio que evalúa la variación intra-específica de la densidad de tricomas con respecto a la presencia de arañas, por lo que se necesitan más estudios para poder generalizar este hallazgo.

En cuanto a los distintos sitios estudiados, si bien variaron en la abundancia de polinizadores y herbívoros, no tenemos evidencia de que esto haya afectado las interacciones estudiadas. La abundancia y riqueza de insectos fue mayor en el sector del humedal que en el resto. Si bien el humedal posee mejores condiciones de humedad también poseen un mayor impacto del ganado, lo que sugiere que la humedad podría estar revirtiendo los potenciales efectos negativos del ganado. No obstante, ninguna de estas diferencias en la abundancia de polinizadores y herbívoros afectó la abundancia de la araña con lo cual su dieta no estaría afectada por la cantidad de alimento. Sería importante llevar a cabo este estudio a lo largo del año para poder determinar cómo la variación en la abundancia de herbívoros, polinizadores y de la araña afectan la adecuación (i.e. frutos/flor) de la planta R. nudiflora a lo largo del tiempo.

Este estudio, a diferencia de otros que analizan sistemas similares, sugiere que la presencia de la araña $P$. viridans no afecta la tasa de visita de polinizadores, los niveles de herbivoría, ni la adecuación de la planta $R$. nudiflora. Esta aparente ausencia de efecto podría deberse a que: en este ambiente, la disponibilidad de alimento parece ser una presión de selección más relevante que el riesgo de depredación; la estimación de riqueza y abundancia de los insectos del lugar fue insuficiente con lo que otorga bajo poder estadístico para detectar efectos; y que las interacciones mutualistas y antagonistas son de similar intensidad y producen un efecto neto nulo. Por ejemplo, el efecto negativo que el ganado puede tener directa o indirectamente sobre las plantas parece compensarse por las mejores características microclimáticas del área donde el ganado es más abundante (Humedal). Adicionalmente, las arañas parecen evitar plantas con una alta densidad de tricomas, lo cual hace revisar el valor adaptativo de estas estructuras. Si las arañas depredaran significativamente sobre los visitantes florales, el valor adaptativo de los tricomas sería doble; por un lado la reducción de la herbivoría por insectos y por otro repeler la presencia de un eventual depredador de sus visitantes florales. Estos resultados resaltan la importancia de estudiar en forma conjunta las interacciones mutualistas y antagonistas para determinar su importancia relativa.

Agradecimientos: A A.G. Farji-Brener y dos revisores anónimos por sus comentarios sobre versiones preliminares de este trabajo. También al Dr. Mahmood Sasa, director de la Estación Palo Verde por su incondicional apoyo. Este estudio fue financiado parcialmente por Beca externa de CONICET (2012-3) y la Organización para Estudios Tropicales.

\section{BIBLIOGRAFÍA}

Аввотт, KR. 2006. Bumblebees avoid flowers containing evidence of past predation events. Canadian Journal of Zoology, 84:1240-1247.

Аввотт, KR. 2010. Background evolution in camouflage systems: A predator-prey/pollinator-flower game. J Theor Biol, 262:662-678.

Arango, AM; Jl Portillo; V Parra-Tabla; LT HernándeZSAlAZAR; JE MORALES-MÁvIL \& V Rico-Gray. 2012. Effect of the spider Peucetia viridans (Oxyopidae) on floral visitors 
and seed set of Cnidoscolus multilobus (Euphorbiaceae). Acta Botanica Mexicana, 100:1-14.

Arango, AM; V Rico-Gray \& V Parra-Tabla. 2000. Population structure, seasonality, and habitat use by the green lynx spider Peucetia viridans (Oxyopidae) inhabiting Cnidoscolus aconitifolius (Euphorbiaceae). J Arachnol, 28:185-194.

BRECHBÜHL, R; C KROPF \& S BACHER. 2010. Impact of flowerdwelling crab spiders on plant-pollinator mutualisms. Basic Appl Ecol, 11:76-82.

DUKAS, R. 2005. Bumble bee predators reduce pollinators density. Ecology, 86:1401-1406.

DukAs, R. \& DH MORSE. 2005. Crab spiders show mixed effects on flower-visiting bees and no effect on plant fitness components. Ecoscience, 12:244-247.

Ebeling, A; A Klein; J Schumacher; W Weisser \& T TsChARNTKE. 2008. How does plant richness affect pollinator richness and temporal stability of flower visits? Oikos, 117:1808-1815.

HARE, JD \& E ElLE. 2002. Variable impact of diverse insect herbivores on dimorphic Datura wrightii. Ecology, 83: 2711-2720.

Higginson, AD; GD Ruxton \& J SKELHORN. 2010. The impact of flower-dwelling predators on host plant reproductive success. Oecologia, 164:411-421.

JANZEN, D. 1991. Historia Natural de Costa Rica. Editorial de la Universidad de Costa Rica, San José, Costa Rica.

Jones, E \& A. Dornhaus. 2011. Predation risk makes bees reject rewarding flowers and reduce foraging activity. Behav Ecol Sociobiol, 65:1505-1511.

KUnIN, WE. 1992. Density and reproductive success in wild populations of Diplotaxis erucoides (Brassicaceae). Oecologia, 91:129-133.

LoudA, SM. 1982. Inflorescence Spiders: A Cost / Benefit Analysis for the Host Plant, Haplopappus venetus Blake (Asteraceae). Oecologia, 55:185-191.

Morais-Filho, JC \& GQ Romero. 2008. Microhabitat use by Peucetia ava (Oxyopidae) on the glandular plant Rhyncanthera dichotoma (Melastomataceae). J Arachnol, 36:374-378.
Morais-FilHo, J.C. \& G.Q. Romero. 2010. Plant glandular trichomes mediate protective mutualism in a spiderplant system. Ecol Entomol, 35:485-494.

Munguía-Rosas, M.; V. Parra-Tabla; J. Ollerton \& J.C. CERVERA. 2012. Environmental control of reproductive phenology and the effect of pollen supplementation on resource allocation in the cleistogamous weed, Ruellia nudi ora (Acanthaceae). Ann Bot, 109:343-50.

Nyffeler, M; DA Dean \& WL Sterling. 1992. Diets, feeding specialization, and predatory role of two lynx spiders, Oxyopes salticus and Peucetia viridans (Araneae: Oxyopidae) in a Texas cotton agroecosystem. Environ Entomol, 21:1457-1465.

Ortegón-Campos, I; L Abdala-Roberts; V Parra-Tabla; JC Cervera; D Marrufo-Zapata \& CM Herrera. 2012. Influence of multiple factors on plant local adaptation: soil type and folivore effects in Ruellia nudiflora (Acanthaceae). Evol Ecol, 26:545-558.

OXFORD, GS \& RG GILLESPIE. 1998. Evolution and ecology of spider coloration. Annu Rev Entomol, 43: 619-643.

Romero, GQ; P Antiqueira \& J Koricheva. 2011. A metaanalysis of predation risk effects on pollinator behaviour. PloS one, 6: e20689.

Romero, GQ; JC SOUZA \& J. VAsconcellos-Neto. 2008. Antiherbivore protection by mutualistic spiders and the role of plant glandular trichomes. Ecology, 89:3105-3115.

Stang, M; PGL Klinkhamer \& E Van Der Meidden. 2006. Size constraints and flower abundance determine the number of interactions in a plant-flower visitor web. Oikos, 112:111-121.

Stevens, M \& S Merilaita. 2009. Animal camouflage: current issues and new perspectives. Philos T Roy Soc B, 364: 423-427.

Vasconcellos-Neto, J. 2006. Associations of spiders of the genus Peucetia (Oxyopidae) with plants bearing glandular hairs. Biotropica, 39:221-226.

Willey, MB \& PH AdLer. 1989. Biology of Peucetia viridans (Araneae, Oxyopidae) in south Carolina, with special reference to predation and maternal care. J Arachnol, 17:275-284. 


\section{INFORMACIÓN SUPLEMENTARIA}

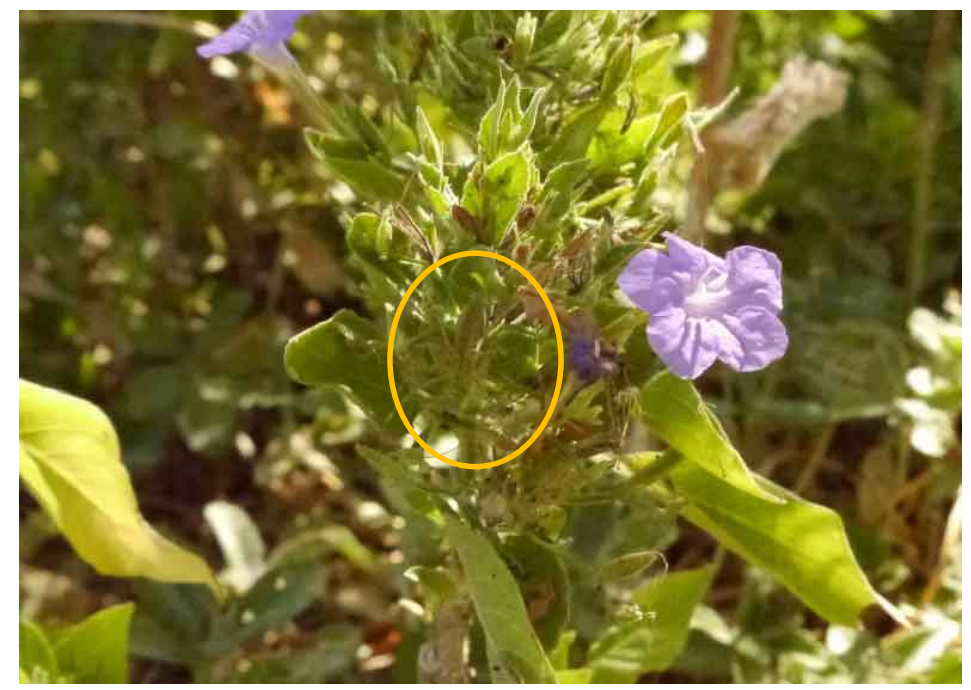

Figura S1. La araña verde, Peucetia viridans (señalada por el óvalo amarillo), camuflada en una planta de Ruellia nudiflora.

Figure S1. The green lynx spider, Peucetia viridans (inside the yellow oval), on the flowers of Ruellia nudiflora. 